\section{Liver stiffness quantification in biopsy- proven nonalcoholic fatty liver disease patients using shear wave elastography in comparison with transient elastography}

\author{
Adele Taibbi', Salvatore Petta², Domenica Matranga ${ }^{3}$, Giovanni Caruana', \\ Roberto Cannella', Gabriele Busè', Vito Di Marco², Massimo Midiri', \\ Tommaso Vincenzo Bartolotta ${ }^{1,4}$
}

'Section of Radiology - BiND, University Hospital "Paolo Giaccone," Palermo; ${ }^{2}$ Section of Gastroenterology and Hepatology, Department of Health Promotion Sciences Maternal and Infant Care, Internal Medicine and Medical Specialties, PROMISE, University of Palermo, Palermo; ${ }^{3}$ Department of Health Promotion Sciences, Maternal and Infant Care, Internal Medicine and Medical Specialties "G. D'Alessandro," University of Palermo, Palermo; ${ }^{4}$ Department of Radiology, Fondazione Istituto Giuseppe Giglio, Cefalù (Palermo), Italy

Purpose: This study prospectively assessed the performance of liver stiffness measurements using point shear-wave elastography ( $p$-SWE) in comparison with transient elastography (TE) in patients with biopsy-proven nonalcoholic fatty liver disease (NAFLD).

Methods: Fifty-six consecutive adult patients with a histological diagnosis of NAFLD prospectively underwent TE and p-SWE on the same day. The median of 10 measurements (SWE-10), the first five (SWE-5), and the first three (SWE-3) measurements were analyzed for p-SWE. Liver biopsy was considered as the reference standard for liver fibrosis grade. Receiver operating characteristic (ROC) curves and areas under the ROC curves (AUROCs) were calculated to assess the performance of TE and p-SWE for the diagnosis of significant (F2-F4) and advanced fibrosis (F3-F4).

Results: Forty-six patients (27 men, 19 women; mean age, 54.7 \pm 9.1 years) had valid p-SWE and TE measurements. Twenty-seven patients (58.7\%) had significant fibrosis and 18 (39.1\%) had advanced fibrosis. For significant fibrosis, both SWE-10 (AUROC, 0.787; $P=0.002$ ) and SWE5 (AUROC, 0.809; $P=0.001$ ) provided higher diagnostic performance than TE (AUROC, 0.719; $P=0.016)$ and SWE-3 (AUROC, 0.714; $P=0.021$ ), albeit without statistical significance $(P=0.301)$. For advanced fibrosis, SWE-5 showed higher diagnostic performance (AUROC, 0.809; $P<0.001$ ) than TE (AUROC, 0.799; $P<0.001$ ), SWE-10 (AUROC, 0.797; $P<0.001$ ), and SWE-3 (AUROC, 0.736; $P=0.003)$, although the differences were not statistically significant $(P=0.496)$. The optimal SWE-10 and SWE- 5 cutoff values were $\geq 8.4$ and $\geq 7.8$ for significant fibrosis, and $\geq 9.1$ and $\geq 8.8$ for advanced fibrosis, respectively.

Conclusion: TE and p-SWE showed similar performance for the diagnosis of significant and advanced fibrosis in NAFLD patients.

Keywords: Nonalcoholic fatty liver disease; Nonalcoholic steatohepatitis; Shear wave elastography; Transient elastography; Liver stiffness

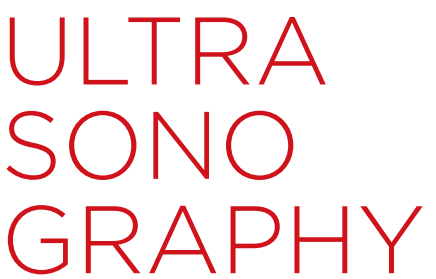

ORIGINAL ARTICLE

https://doi.org/10.14366/usg.20147 pISSN: 2288-5919 - elSSN: 2288-5943 Ultrasonography 2021;40:407-416

Received: September 18, 2020

Revised: December 17, 2020

Accepted: December 18, 2020

Correspondence to:

Roberto Cannella, MD, Section of Radiology - BiND, University Hospital

"Paolo Giaccone," Via del Vespro 129, 90127 Palermo, Italy

Tel. +39-0916552335

Fax. $+39-0916552381$

E-mail: rob.cannella89@gmail.com

This is an Open Access article distributed under the terms of the Creative Commons Attribution NonCommercial License (http://creativecommons.org/ licenses/by-nc/4.0/) which permits unrestricted noncommercial use, distribution, and reproduction in any medium, provided the original work is properly cited.

Copyright @ 2021 Korean Society of Ultrasound in Medicine (KSUM)

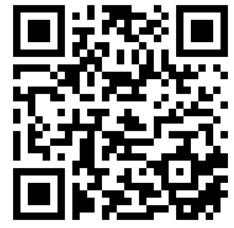

How to cite this article:

Taibbi A, Petta S, Matranga D, Caruana G Cannella $R$, Busè $G$, et al. Liver stiffness quantification in biopsy-proven nonalcoholic fatty liver disease patients using shear wave elastography in comparison with transient elastography. Ultrasonography. 2021 Jul:40(3):407-416. 


\section{Introduction}

Diffuse liver diseases have recently received increasing consideration due to their mounting incidence, with particular attention to hepatic steatosis [1], which is related to two main clinical-pathological entities: alcoholic fatty liver disease and nonalcoholic fatty liver disease (NAFLD). NAFLD affects approximately $90 \%$ of the obese population, as well as $15 \%-40 \%$ of the general population. NAFLD can result in a multistep progressive disease, potentially evolving into nonalcoholic steatohepatitis (NASH), advanced fibrosis (in approximately $25 \%$ of cases), cirrhosis ( $10 \%-20 \%$ of cases), and hepatocellular carcinoma (4\% of cases) [2-4]. In NAFLD patients, the most important clinical end-points are the differentiation of NASH from simple hepatic steatosis and the identification of advanced fibrosis. Considering the increased risk of liver failure and hepatocellular carcinoma in patients with cirrhosis, it is of the utmost relevance to follow-up fibrosis evolution noninvasively in order to establish an appropriate treatment and avoid disease progression [5].

The reference standard for the diagnosis of hepatic fibrosis is liver biopsy, an invasive procedure with known complications that is prone to sampling errors, intra- and inter-observer variability, and limited repeatability. Among the various noninvasive methods as alternative tools for staging hepatic fibrosis, the currently mostused technique in clinical practice is transient elastography (TE; FibroScan, Echosens, Paris, France). However, this technique has some limitations, such as obesity (which was partially overcome by the introduction of a new XL probe), narrow intercostal spaces, and the presence of ascites. When using the conventional $\mathrm{M}$ probe, in patients with steatosis of $>66 \%$ at liver biopsy, TE provides a higher false-positive rate in terms of liver stiffness (LS) measurements. Therefore, TE (using the $\mathrm{M}$ probe) may overestimate liver fibrosis in patients with a body mass index (BMI) $>30 \mathrm{~kg} / \mathrm{m}^{2}$ and severe steatosis [6].

Point shear-wave elastography ( $p$-SWE) may represent an alternative to TE. SWE is integrated into conventional ultrasound equipment, enabling a rapid quantitative evaluation of LS [7]. SWE allows the operator to precisely visualize where the measurements are performed on B-mode ultrasound images, even in presence of ascites [8]. Preliminary results have shown high accuracy of $p$-SWE for the assessment of liver fibrosis in patients affected by chronic viral hepatitis [9-11], but few data are available regarding the comparison between $\mathrm{p}$-SWE and TE, especially in patients with NAFLD $[12,13]$ and to our knowledge, no prior study has assessed NAFLD using an RS80A ultrasound system. Therefore, in the last version of the European Federation of Societies for Ultrasound in Medicine and Biology (EFSUMB) guidelines on the clinical use of liver ultrasound elastography, recommendations are still lacking on the preferred first-line method for the assessment of hepatic fibrosis in patients with NAFLD [14].

The aim of our study was to prospectively assess the accuracy of p-SWE for the quantitative evaluation of LS in patients with NAFLD, in comparison with TE and liver biopsy, and to determine the optimal cutoff values for significant and advanced fibrosis.

\section{Materials and Methods}

This prospective study was approved by the Ethics Committee of University Hospital, a tertiary center for the treatment of chronic liver disease (Palermo 1 ID-2014). All subjects provided written informed consent for this study in accordance with the Declaration of Helsinki.

\section{Population}

Between March 2017 and November 2018, 56 consecutive adult patients with a histological diagnosis of NAFLD prospectively underwent TE (FibroScan, Echosens) and p-SWE (RS80A ultrasound system, Samsung Medison, Seoul, Korea) on the same day. The following exclusion criteria were considered: (1) a history of significant alcohol consumption (>21 standard drinks per week in men or $>14$ standard drinks per week in women) or virus-related chronic hepatitis; (2) a history of taking drugs that may cause steatosis, obstructive cholestasis, acute hepatitis, and infiltrative liver diseases, which may affect the reliability of LS measurements; (3) liver inflammation, as indicated by aspartate transaminase (AST) and/or alanine transaminase (ALT) elevation $>5$ times the normal limits. In addition, after performing p-SWE, 10 (17.8\%) patients were excluded due to failure of more than $75 \%$ of the SWE measurements considering a qualitative numeric index (the Reliability Measurement Index [RMI]) automatically calculated by the SWE software and shown on the ultrasonography (US) device screen.

The same day of TE and p-SWE examinations, laboratory markers including ALT (IU/L), AST (IU/L), total cholesterol $(\mathrm{mg} / \mathrm{dL})$, and triglycerides $(\mathrm{mg} / \mathrm{dL})$, as well as BMI $\left(\mathrm{kg} / \mathrm{m}^{2}\right)$, were collected.

\section{Imaging Techniques}

All patients underwent TE performed with the FibroScan (Echosens) using the $\mathrm{M}$ or $\mathrm{XL}$ probe (46 and 10 patients, respectively). LS was assessed after at least 4 hours of fasting by a trained operator with more than 15 years of experience who had previously performed at least 300 examinations in patients with chronic liver disease. The probe was positioned at the level of the right liver lobe through the intercostal spaces with patients in the supine position with the right arm in maximum abduction. TE displayed the region of 
interest (ROI) in ultrasound A-mode and measured LS in a volume represented by a cylinder $1 \mathrm{~cm}$ wide and $4 \mathrm{~cm}$ long located between $25 \mathrm{~mm}$ and $65 \mathrm{~mm}$ below the skin surface. Uncorrected acquisitions are automatically ruled out from the US software. The results were expressed in kilopascals ( $\mathrm{KPa}$ ) and ranged from 2.5 to $75 \mathrm{kPa}$.

The most important parameter for assessing the reliability of $T E$ evaluation is the interquartile range (IQR), which reflects the variability of the validated measures, and should not exceed $20 \%$ $30 \%$ of the median value. All LS measurements were classified into three reliability categories: (1) very reliable (IQR/M $\leq 0.10)$; (2) reliable $(0.10<\mathrm{IQR} / \mathrm{M} \leq 0.30$, or IQR/M $>0.30$ with $\mathrm{LS}$ median $<7.1$ $\mathrm{kPa})$; or (3) poorly reliable (IQR/M $>0.30$ with $\mathrm{LS}$ median $\geq 7.1 \mathrm{kPa})$. Only patients with 10 valid measurements were included, and poorly reliable results were excluded from the analysis, without being further enrolled for SWE [15].

All p-SWE examinations were performed by one radiologist, blinded to biopsy and laboratory results, using an ultrasound machine (Samsung RS80A, Samsung Medison Co.) equipped with p-SWE software on a convex broadband probe (CA1-7A). All patients fasted for a minimum of 2 hours and rested for a minimum of 10 minutes before undergoing $p$-SWE evaluation. During the LS measurements, the probe was positioned on the right liver lobe, through the intercostal spaces with the patient lying in supine position with the right arm in maximal abduction. LS measurements were obtained as median values in kilopascals $(\mathrm{kPa})$ from a rectangular ROI placed between $2 \mathrm{~cm}$ from the liver capsule and at a maximum depth of up to $5 \mathrm{~cm}$ beneath the skin in the hepatic parenchyma, carefully avoiding large intrahepatic vessels and biliary structures, and positioning the focus at the level of the ROI. During the LS measurements, patients were asked to stop breathing without a deep inspiration. For each measurement, the US equipment provided the RMI as an indicator of the calculated stiffness reliability, with a maximum value of 1 . The US manufacturer developed this performance index through phantom and in vivo studies, since elastography data are often impaired by low signalto-noise ratio, making the results unreliable. It is obtained by the weighted sum of the residual of the wave equation and the magnitude of the shear wave. Pre-established RMI cutoff values are highly correlated with reproducible measurements and can be utilized to rule out unreliable measurements, thereby improving shear wave elastography performance [16]. Only measurements with an RMI $\geq 0.6$ and IQR/M ratios $\leq 30 \%$ were considered acceptable. Ten consecutive reliable measurements expressed in $\mathrm{kPa}$ were acquired in this study.

\section{Reference Standard}

Liver biopsy was considered as the reference standard and was performed in all patients within 1 month in order to confirm the final diagnosis of NAFLD/NASH and to grade liver fibrosis. All biopsy specimens were evaluated by a single experienced pathologist, who was blinded to the patients' clinical and LS results. Hepatic fibrosis was graded according to the Kleiner score for NAFLD, as follows: F0, absence of fibrosis; F1, perisinusoidal or periportal fibrosis; F2, perisinusoidal and portal/periportal fibrosis; $\mathrm{F}$, septal or bridging fibrosis; and F4, cirrhosis [17]. The NAFLD activity score (NAS), grade of steatosis $(0-3)$, lobular inflammation (0-3), and hepatocyte ballooning (0-2) were also recorded for each patient [18]. For the purposes of this study, grades F2-F4 were defined as significant fibrosis, while grades F3-F4 were defined as advanced fibrosis.

\section{Statistical Analysis}

The statistical analysis was performed by an expert statistician, in order to (1) compare patients with valid and failed p-SWE; (2) assess differences in diagnostic performances using p-SWE with 10 measurements (SWE-10), the first five (SWE-5), and the first three (SWE-3) LS measurements; (3) calculate optimal TE and p-SWE cutoffs for the diagnosis of significant (F2-F3) and advanced (F3F4) fibrosis; and (4) correlate TE and p-SWE measurements with histopathological and laboratory markers.

Continuous variables were expressed as mean and standard deviation. Categorical variables were expressed as numbers and percentages. Continuous variables were compared using analysis of variance and the independent $t$ test, while categorical variables were compared using the chi-square test. Receiver operating characteristic (ROC) curves with 95\% confidence intervals (Cls) and areas under the ROC curve (AUROCs with $95 \% \mathrm{Cls}$ ) were calculated to assess the diagnostic performance of successful TE and p-SWE measurements for the diagnosis of significant and advanced fibrosis. AUROCs for TE and p-SWE were compared using the DeLong test. Optimal cutoff values based on the Youden index of sensitivity and specificity, as well as alternative cutoffs for sensitivity $\geq 90 \%$ and specificity $\geq 90 \%$ were calculated. The correlations between TE, p-SWE, fibrosis, histopathological features of NASH (i.e., NAS, steatosis, inflammation, and ballooning), and laboratory markers (i.e., total cholesterol, triglycerides, AST, and ALT) was evaluated using the Spearman rank correlation coefficient (Spearman rho).

The statistical significance level was set at $P<0.05$, except for the Spearman rho, for which it was set at $\mathrm{P}<0.01$ due to multiple comparisons. The statistical analysis was conducted using SPSS software version 20.0 (IBM Corp., Armonk, NY, USA). 


\section{Results}

\section{Population Characteristics}

The demographic and histopathological characteristics in the study population are summarized in Table 1. The final study population

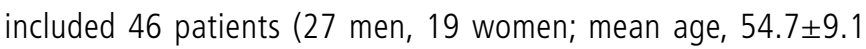
years; range, 40 to 73 years) with NAFLD. Patients' BMI ranged from 21.2 to $40.4 \mathrm{~kg} / \mathrm{m}^{2}$ (mean value, $29.4 \pm 4.5 \mathrm{~kg} / \mathrm{m}^{2}$ ). Diabetes was present in 18 patients (39.1\%), whereas four patients (8.7\%) had impaired fasting glycemia. Eleven patients $(23.9 \%)$ presented cholesterol $>200 \mathrm{mg} / \mathrm{dL}$ and six patients (13.0\%) had triglyceride levels $>175 \mathrm{mg} / \mathrm{dL}$.

Through percutaneous liver biopsy, hepatic fibrosis was graded as F0 in four cases (8.7\%), F1 in 15 cases (32.6\%), F2 in nine cases

Table 1. Baseline characteristics of the study population

\begin{tabular}{|c|c|c|c|}
\hline & $\begin{array}{l}\text { Total } \\
(n=46)\end{array}$ & $\begin{array}{c}\text { Significant } \\
\text { fibrosis }(n=27)\end{array}$ & $\begin{array}{c}\text { Advanced } \\
\text { fibrosis }(n=18)\end{array}$ \\
\hline \multicolumn{4}{|l|}{ Sex } \\
\hline Male & $27(58.7)$ & $16(40.7)$ & $10(55.6)$ \\
\hline Female & $19(41.3)$ & $11(59.3)$ & $8(44.4)$ \\
\hline Age (year) & $54.7 \pm 9.1$ & $57.9 \pm 9.0$ & $58.8 \pm 8.2$ \\
\hline $\mathrm{BMI}\left(\mathrm{kg} / \mathrm{m}^{2}\right)$ & $29.4 \pm 4.5$ & $29.8 \pm 5.1$ & $30.0 \pm 4.9$ \\
\hline \multicolumn{4}{|l|}{ Fibrosis } \\
\hline F0 & $4(8.7)$ & 0 & 0 \\
\hline $\mathrm{F} 1$ & $15(32.6)$ & 0 & 0 \\
\hline $\mathrm{F} 2$ & $9(19.6)$ & $9(33.3)$ & 0 \\
\hline F3 & $5(10.9)$ & $5(18.5)$ & $5(27.8)$ \\
\hline $\mathrm{F} 4$ & $13(28.2)$ & $13(48.2)$ & $13(72.2)$ \\
\hline NAS & $4.3 \pm 1.5$ & $4.6 \pm 1.6$ & $4.3 \pm 1.6$ \\
\hline \multicolumn{4}{|l|}{ Steatosis grade } \\
\hline $0(<5 \%)$ & $1(2.2)$ & $1(3.7)$ & $1(5.6)$ \\
\hline $1(5 \%-33 \%)$ & $14(30.4)$ & $8(29.7)$ & $7(38.9)$ \\
\hline $2(33 \%-66 \%)$ & $17(37.0)$ & $9(33.3)$ & $4(22.2)$ \\
\hline $3(>66 \%)$ & $14(30.4)$ & $9(33.3)$ & $6(33.3)$ \\
\hline \multicolumn{4}{|l|}{ Inflammation } \\
\hline 0 (no foci) & $2(4.4)$ & $1(3.7)$ & $1(5.6)$ \\
\hline 1 (<2 foci per ×200) & $20(43.4)$ & $7(25.9)$ & $5(27.7)$ \\
\hline 2 (2-4 foci per $\times 200)$ & $22(47.8)$ & $17(63.0)$ & $11(61.1)$ \\
\hline 3 (>4 foci per $\times 200$ ) & $2(4.4)$ & $2(7.4)$ & $1(5.6)$ \\
\hline \multicolumn{4}{|l|}{ Ballooning } \\
\hline 0 (none) & $15(32.6)$ & $7(25.9)$ & $6(33.3)$ \\
\hline 1 (few balloon cells) & $26(56.5)$ & $16(59.3)$ & $10(55.6)$ \\
\hline 2 (many cells) & $5(10.9)$ & $4(14.8)$ & $2(11.1)$ \\
\hline
\end{tabular}

Values are presented as number (\%) or mean \pm standard deviation.

BMI, body mass index; NAS, nonalcoholic fatty liver disease activity score.
(19.6\%), F3 in five cases (10.9\%), and F4 in the remaining 13 cases (28.2\%). Overall, significant fibrosis (F2-F4) was present in 27 patients (58.7\%), while advanced fibrosis (F3-F4) was observed in 18 patients (39.1\%).

None of the patients had poorly reliable TE evaluations, whereas $10(17.8 \%)$ subjects were excluded from the initial population due to failure of more than $75 \%$ of the SWE measurements. The excluded subjects had advanced (F3-F4) or significant (F2) fibrosis in nine cases $(90.0 \%)$ and one case $(10.0 \%)$, respectively. Compared to patients with successful p-SWE LS measurements, those with failed $p$-SWE measurements more frequently had advanced fibrosis $(P=0.006)$, as well as having a higher BMI (mean, $33.7 \pm 4.9 \mathrm{~kg} / \mathrm{m}^{2}$; $\mathrm{P}=0.011$ ), and higher TE LS measurements (mean, $28.9 \pm 25.4 \mathrm{kPa}$; $\mathrm{P}<0.001)$.

\section{Diagnostic Performance of TE and SWE}

The diagnostic performance of TE and SWE is presented in Table 2. For the diagnosis of significant fibrosis, both SWE-10 (AUROC, 0.787; $95 \% \mathrm{Cl}, 0.646$ to $0.927 ; \mathrm{P}=0.002$ ) and SWE-5 (AUROC, 0.809; $95 \% \mathrm{Cl}, 0.676$ to $0.942 ; \mathrm{P}=0.001)$ provided higher diagnostic performance than TE (AUROC, $0.719 ; 95 \% \mathrm{Cl}, 0.572$ to 0.867 ; $\mathrm{P}=0.016$ ) and SWE-3 (AUROC, $0.714 ; 95 \% \mathrm{Cl}, 0.560$ to 0.869 ; $P=0.021$ ), although the differences among AUROC curves were not statistically significant $(P=0.301)$ (Figs. 1, 2).

For the diagnosis of advanced fibrosis, SWE- 5 showed a higher diagnostic performance (AUROC, $0.809 ; 95 \% \mathrm{Cl}, 0.684$ to 0.933 ;

Table 2. Diagnostic performance of TE and SWE-10, SWE-5, or SWE-3 for the diagnosis of significant (F2-F4) and advanced fibrosis (F3-F4)

\begin{tabular}{lccc}
\hline & AUROC & $95 \% \mathrm{Cl}$ & P-value \\
\hline Significant fibrosis & & & \\
TE & 0.719 & $0.572-0.867$ & 0.016 \\
SWE-10 & 0.787 & $0.646-0.927$ & 0.002 \\
SWE-5 & 0.809 & $0.676-0.942$ & 0.001 \\
SWE-3 & 0.714 & $0.560-0.869$ & 0.021 \\
P-value & 0.301 & & \\
Advanced fibrosis & & & \\
TE & 0.799 & $0.646-0.952$ & $<0.001$ \\
SWE-10 & 0.797 & $0.659-0.935$ & $<0.001$ \\
SWE-5 & 0.809 & $0.684-0.933$ & $<0.001$ \\
SWE-3 & 0.736 & $0.587-0.885$ & 0.003 \\
P-value & 0.496 & &
\end{tabular}

TE, transient elastography; SWE-10, p-SWE with 10 measurements; SWE-5, p-SWE with five measurements; SWE-3, p-SWE with three measurements; $p$-SWE, point shear-wave elastography; $A U R O C$, area under the receiving operator characteristic curve; $\mathrm{Cl}$, confidence interval. 
$\mathrm{P}<0.001)$ than TE (AUROC, $0.799 ; 95 \% \mathrm{Cl}, 0.646$ to 0.952 ; $\mathrm{P}<0.001$ ), SWE-10 (AUROC, $0.797 ; 95 \% \mathrm{Cl}, 0.659$ to 0.935 ; $\mathrm{P}<0.001$ ), and SWE-3 (AUROC, $0.736 ; 95 \% \mathrm{Cl}, 0.587$ to 0.885 ; $\mathrm{P}=0.003)$, although the differences among AUROC curves did not reach statistical significance ( $P=0.496)$ (Figs. 3, 4).

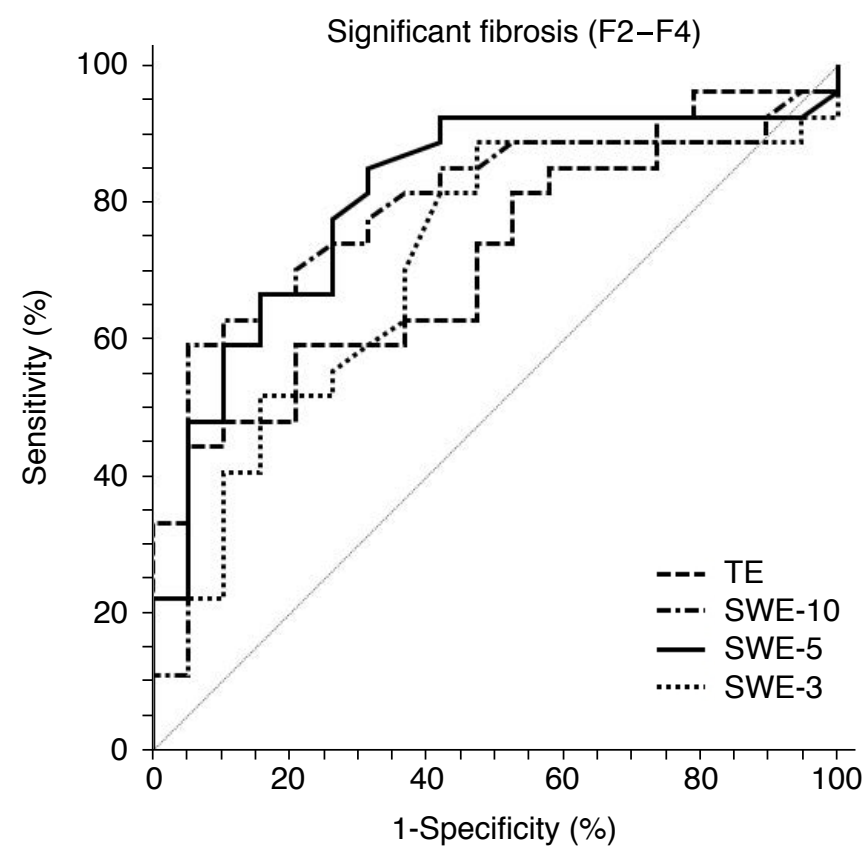

Fig. 1. Receiver operating characteristic curves for liver stiffness measurements obtained with transient elastography (TE) and point shear-wave elastography ( $p$-SWE) for the diagnosis of significant fibrosis (F2-F4). SWE-10, p-SWE with 10 measurements; SWE-5, p-SWE with five measurements; SWE-3, p-SWE with three measurements.

\section{Optimal Cutoff for the Diagnosis of Significant and Advanced} Fibrosis

The optimal cutoff values based on the Youden index of sensitivity and specificity are reported in Table 3. When using $\mathrm{TE}$, an $\mathrm{LS} \geq 7.9$ $\mathrm{kPa}$ demonstrated a sensitivity of $63.0 \%$ and a specificity of $63.2 \%$

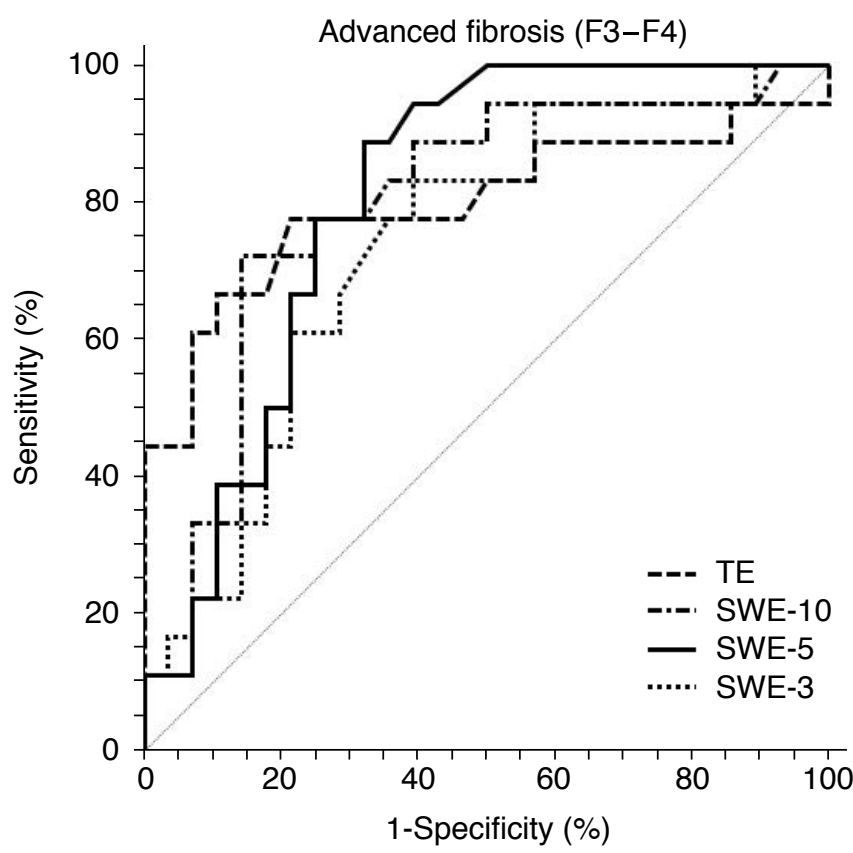

Fig. 3. Receiver operating characteristic curve for liver stiffness measurements obtained with transient elastography (TE) and point shear-wave elastography ( $p$-SWE) for the diagnosis of advanced fibrosis (F3-F4). SWE-10, p-SWE with 10 measurements; SWE-5, p-SWE with five measurements; SWE-3, p-SWE with three measurements.

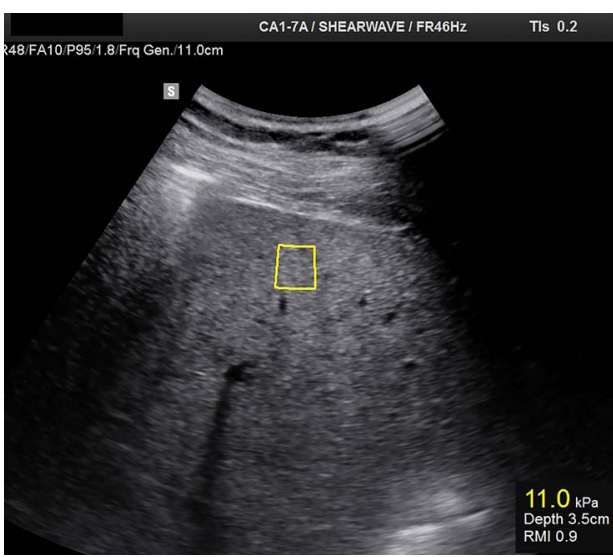

A

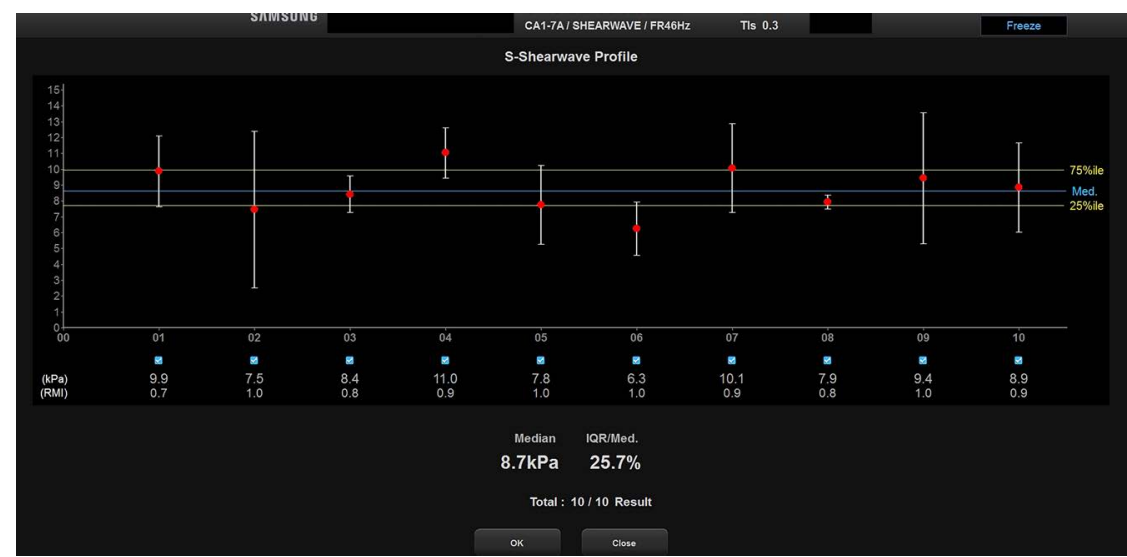

B

Fig. 2. Point shear-wave elastography in a 44-year-old man with significant fibrosis.

A. Intercostal ultrasound image in the supine position shows a region of interest located under the liver capsule at a depth of $3.5 \mathrm{~cm}$. B. Ten valid measurements are reported with a final median liver stiffness value of $8.7 \mathrm{kPa}$ indicated in the lowest part of the image. A good interquartile range is also shown. 
for the diagnosis of significant fibrosis, while an $L S \geq 8.5 \mathrm{kPa}$ had a sensitivity of $77.8 \%$ and a specificity of $78.6 \%$ for the diagnosis of advanced fibrosis. When using the conventional SWE-10, an LS of $\geq 8.4 \mathrm{kPa}$ demonstrated a sensitivity of $74.0 \%$ and a specificity of $73.7 \%$ for the diagnosis of significant fibrosis (Fig. 2), while an LS $\geq 9.1 \mathrm{kPa}$ had a sensitivity of $72.2 \%$ and a specificity of $78.5 \%$ for the diagnosis of advanced fibrosis (Fig. 4). A slight increase in sensitivity, without a significant loss in specificity, was noted for the SWE- 5 cutoffs of $\geq 7.8 \mathrm{kPa}$ for the diagnosis of significant fibrosis and $\geq 8.8 \mathrm{kPa}$ for the diagnosis of advanced fibrosis (sensitivity of $77.8 \%$ with both cutoffs).

Alternative cutoffs calculated in order to achieve the highest sensitivity and specificity $(\geq 90 \%)$ are presented in Table 4 . When using TE, an alternative cutoff of $4.6 \mathrm{kPa}$ increased the sensitivity of the diagnosis of advanced fibrosis to $94.4 \%$, with a significant drop in specificity to $14.3 \%$. Interestingly, an alternative cutoff of 7.6 $\mathrm{kPa}$ for SWE-5 increased the sensitivity to $94.4 \%$, with little drop in specificity $(60.7 \%)$ for the diagnosis of advanced fibrosis.

\section{Correlations of LS Measurements with Clinical and Histopathological Parameters}

Table 5 shows the correlations between LS measurements measured with TE and $\mathrm{p}$-SWE and fibrosis grade, histopathological markers of $\mathrm{NASH}$, and selected laboratory parameters. There were statistically significant correlations between TE and SWE-10 ( $P=0.001)$, SWE-5 ( $P=0.001)$, and SWE-3 ( $P=0.006)$. Statistically significant correlations were likewise observed between fibrosis grade and TE $(P<0.001)$, SWE-10 $(P<0.001)$, SWE-5 $(P<0.001)$, and SWE-3 $(P=0.005)$. LS measurements were significantly different in patients with and without diabetes using TE ( $P=0.006)$, SWE-10 ( $P=0.006)$, and SWE-5 ( $P=0.016)$, but not using SWE-3 $(P=0.125)$.

\section{Discussion}

Our study demonstrated that p-SWE provided accurate results for the assessment of hepatic fibrosis in patients with biopsyproven NAFLD, with a fair-to-good performance for the diagnosis of significant and advanced fibrosis. Compared to $T E$, there were

Table 3. Cutoff values based on the Youden index, with sensitivity and specificity values, of liver stiffness measurement by TE and SWE-10, SWE-5, or SWE-3 for the diagnosis of significant (F2-F4) and advanced fibrosis (F3-F4)

\begin{tabular}{lcccc}
\hline & $\begin{array}{c}\text { Cutoff } \\
(\mathrm{kPa})\end{array}$ & $\begin{array}{c}\text { Sensitivity } \\
(\%)\end{array}$ & $\begin{array}{c}\text { Specificity } \\
(\%)\end{array}$ & $\begin{array}{c}\text { Accuracy } \\
(\%)\end{array}$ \\
\hline Significant fibrosis & & & & \\
TE & $\geq 7.9$ & 63.0 & 63.2 & 63.0 \\
SWE-10 & $\geq 8.4$ & 74.0 & 73.7 & 73.9 \\
SWE-5 & $\geq 7.8$ & 77.8 & 73.4 & 76.1 \\
SWE-3 & $\geq 7.8$ & 66.7 & 63.2 & 65.2 \\
Advanced fibrosis & & & & \\
TE & $\geq 8.5$ & 77.8 & 78.6 & 78.3 \\
SWE-10 & $\geq 9.1$ & 72.2 & 78.5 & 76.1 \\
SWE-5 & $\geq 8.8$ & 77.8 & 75.0 & 76.1 \\
SWE-3 & $\geq 8.2$ & 66.7 & 71.4 & 69.6 \\
\hline
\end{tabular}

TE, transient elastography; SWE-10, p-SWE with 10 measurements; SWE-5, p-SWE with five measurements; SWE-3, p-SWE with three measurements; $p$-SWE, point shear-wave elastography.

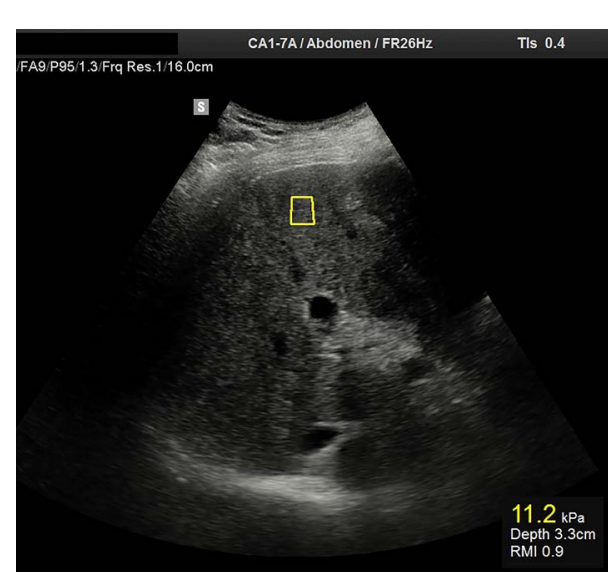

A

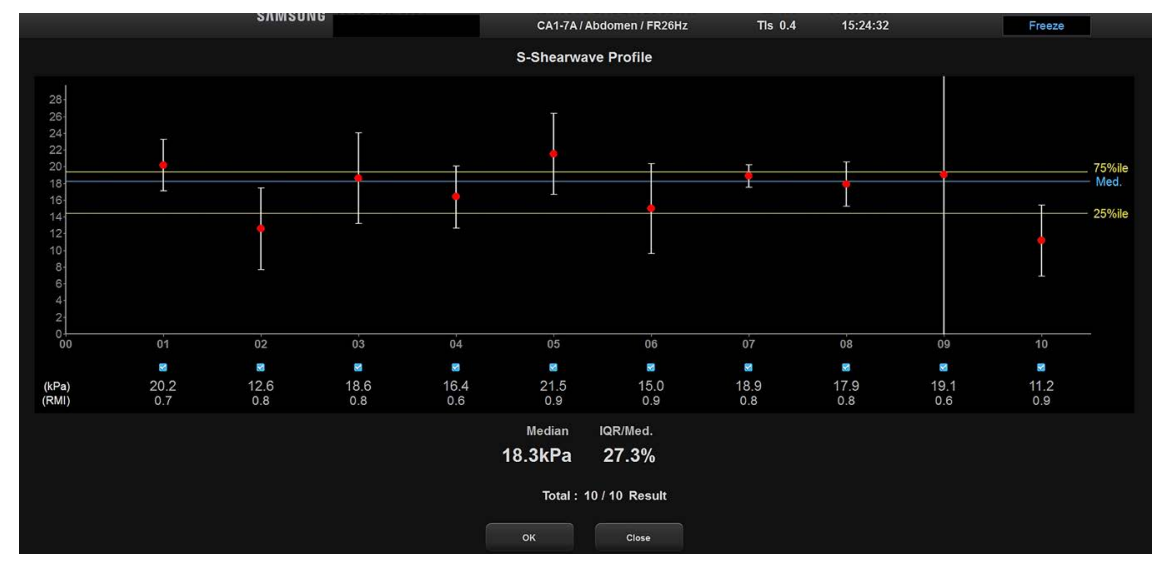

B

Fig. 4. Point shear-wave elastography in a 69-year-old man with advanced fibrosis.

A. Intercostal ultrasound image in the supine position shows a region of interest located under the liver capsule at a depth of $3.3 \mathrm{~cm}$. B. Ten valid measurements are reported with a final median liver stiffness value of $18.3 \mathrm{kPa}$, as indicated in the lowest part of the image. A good interquartile range is also shown. 
Table 4. Alternative cutoffs calculated in order to achieve high sensitivity or specificity ( $\geq 90 \%$ ) of liver stiffness measurements by TE and SWE-10, SWE-5, or SWE-3 for the diagnosis of significant (F2-F4) and advanced fibrosis (F3-F4)

\begin{tabular}{lcccc}
\hline \multicolumn{1}{c}{ Exam } & Aim & Cutoff $(\mathrm{kPa})$ & Sensitivity (\%) & Specificity (\%) \\
\hline Significant fibrosis & & & & \\
TE & Sensitivity $\geq 90 \%$ & 4.8 & 92.6 & 26.3 \\
SWE-10 & Specificity $\geq 90 \%$ & 11.1 & 44.4 & 94.7 \\
& Sensitivity $\geq 90 \%$ & 5.5 & 92.6 & 10.5 \\
SWE-5 & Specificity $\geq 90 \%$ & 9.3 & 59.3 & 94.7 \\
& Sensitivity $\geq 90 \%$ & 6.9 & 92.6 & 57.9 \\
SWE-3 & Specificity $\geq 90 \%$ & 9.9 & 48.2 & 94.7 \\
& Sensitivity $\geq 90 \%$ & 5.3 & 92.6 & 5.26 \\
Advanced fibrosis & Specificity $\geq 90 \%$ & 13.0 & 22.2 & 94.7 \\
TE & & & & \\
& Sensitivity $\geq 90 \%$ & 4.6 & 94.4 & 14.3 \\
SWE-10 & Specificity $\geq 90 \%$ & 11.1 & 61.1 & 92.9 \\
& Sensitivity $\geq 90 \%$ & 6.9 & 94.4 & 50.0 \\
SWE-5 & Specificity $\geq 90 \%$ & 12.5 & 33.3 & 92.9 \\
SWE-3 & Sensitivity $\geq 90 \%$ & 7.6 & 94.4 & 60.7 \\
& Specificity $\geq 90 \%$ & 13.8 & 22.2 & 92.9 \\
\hline
\end{tabular}

TE, transient elastography; SWE-10, p-SWE with 10 measurements; SWE-5, p-SWE with five measurements; SWE-3, p-SWE with three measurements; p-SWE, point shear-wave elastography.

Table 5. Correlations between TE, p-SWE, fibrosis grade, histopathological features, and laboratory markers

\begin{tabular}{|c|c|c|c|c|c|c|c|c|c|c|c|}
\hline Exam & TE & Fibrosis & NAS & Steatosis & Inflam. & Ballooning & BMI & Chol. & TG & AST & ALT \\
\hline TE & - & $0.582^{a)}$ & 0.210 & 0.176 & 0.108 & 0.177 & 0.216 & -0.275 & -0.009 & 0.232 & -0.082 \\
\hline P-value & - & $<0.001^{\mathrm{a})}$ & 0.161 & 0.242 & 0.473 & 0.239 & 0.150 & 0.010 & 0.959 & 0.168 & 0.161 \\
\hline SWE-10 & $0.461^{\text {a) }}$ & $0.528^{a)}$ & 0.179 & 0.063 & 0.290 & 0.119 & -0.084 & -0.214 & 0.084 & 0.137 & 0.179 \\
\hline P-value & $0.001^{a)}$ & $<0.001^{\mathrm{a})}$ & 0.235 & 0.680 & 0.050 & 0.436 & 0.956 & 0.203 & 0.620 & 0.420 & 0.235 \\
\hline SWE-5 & $0.466^{a)}$ & $0.567^{\mathrm{a})}$ & 0.286 & 0.158 & 0.286 & 0.246 & 0.046 & -0.295 & 0.145 & -0.017 & 0.158 \\
\hline P-value & $0.001^{a)}$ & $<0.001^{\text {a) }}$ & 0.054 & 0.294 & 0.054 & 0.099 & 0.769 & 0.077 & 0.402 & 0.922 & 0.294 \\
\hline SWE-3 & $0.403^{\mathrm{a})}$ & $0.404^{a)}$ & 0.126 & 0.075 & 0.079 & 0.157 & 0.015 & -0.182 & -0.083 & -0.002 & 0.075 \\
\hline P-value & $0.006^{\mathrm{a})}$ & $0.005^{\mathrm{a})}$ & 0.403 & 0.622 & 0.600 & 0.298 & 0.924 & 0.282 & 0.627 & 0.993 & 0.622 \\
\hline
\end{tabular}

Numbers represent the Spearman rank correlation coefficient (rho).

$\mathrm{TE}$, transient elastography; p-SWE, point shear-wave elastography; NAS, nonalcoholic fatty liver disease activity score; Inflam, inflammation; BMI, body mass index; Chol., cholesterol; TG, triglycerides; AST, aspartate transaminase; ALT, alanine transaminase; SWE-10, p-SWE with 10 measurements; SWE-5, p-SWE with five measurements; SWE-3, $\mathrm{p}$-SWE with three measurements.

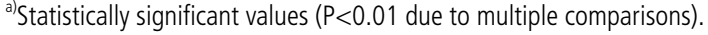

no significant differences in diagnostic performances of SWE-10, SWE-5, or SWE-3 for the diagnosis of both significant $(P=0.301)$ and advanced $(P=0.496)$ fibrosis, although SWE-10 and SWE5 had higher performance. Moreover, TE and different $p$-SWE measurements showed significant correlations with the fibrosis grade evaluated on biopsy specimens. Considering the widespread prevalence of NAFLD in the general population related to the increased prevalence of obesity and metabolic syndrome, our results may have significant relevance for the appropriate firstline noninvasive assessment of patients with NAFLD. Indeed, the presence of significant and advanced fibrosis is an independent predictor of liver-related mortality, and has been associated with an increased risk of hepatocellular carcinoma, cardiovascular mortality, and type 2 diabetes mellitus [19]. 
Limited literature exists comparing different methods for LS measurements in the same study population affected by NAFLD, especially in comparison with biopsy results $[12,17,20,21]$. Our results are concordant with those of Furlan et al. [20], who reported comparable diagnostic accuracy of SWE to conventional TE for the identification of significant and advanced fibrosis in NAFLD patients. In our study, the optimal cutoffs of $\geq 8.4 \mathrm{kPa}$ and $\geq 9.1 \mathrm{kPa}$ obtained by SWE-10 provided a sensitivity of $74.0 \%$ and a specificity of $73.7 \%$ for the diagnosis of significant fibrosis, and a sensitivity of $72.2 \%$ and a specificity of $78.5 \%$ for the diagnosis of advanced fibrosis, respectively. This evidence supports the use of p-SWE in clinical practice as a valuable alternative noninvasive method for the quantification of $L S$ and a potential alternative to liver biopsy for the staging and follow-up of patients with NAFLD. SWE may have advantages over TE in patients with ascites or obesity, and may enable a simultaneous evaluation of focal liver lesions and LS measurements in patients undergoing surveillance for NAFLD [6].

Moreover, our study assessed the performance of the $p$-SWE technique with fewer measurements. In agreement with other recent evidence evaluating cohorts with different etiologies of chronic liver disease, we observed that SWE-5 provided the highest diagnostic performance (AUROC), sensitivity, and specificity for both significant $(0.809,77.8 \%$, and $73.4 \%$, respectively) and advanced fibrosis $(0.809,77.8 \%$, and $75.0 \%$, respectively) with respect to SWE-10 and for significant fibrosis with respect to TE, even if the differences were not statistically significant $[22,23]$. Interestingly, when exploring alternative cutoffs for maximizing sensitivity and specificity, SWE-5 with a cutoff of $\geq 7.6 \mathrm{kPa}$ yielded an increase in sensitivity to $94.4 \%$, with only a slight drop in specificity (60.7\%) for the diagnosis of advanced fibrosis. In contrast, SWE-3 showed the lowest performance (AUROC, sensitivity, and specificity) for both significant $(0.714,66.7 \%$, and $63.2 \%$, respectively) and advanced fibrosis $(0.736,66.7 \%$, and $71.4 \%$, respectively). Although current guidelines still recommend a minimum of 10 valid measurements for the appropriate p-SWE examinations, conducting an evaluation with five valid LS measurements may potentially save time during examinations in clinical practice, without compromising the diagnostic performance for the assessment of hepatic fibrosis [14].

In our study, LS measurements by TE and p-SWE showed significant correlations with fibrosis grade, while no correlations were observed for BMI, histopathological markers of $\mathrm{NASH}$, or laboratory results. However, in a non-negligible proportion of patients (17.8\% of the initial cohort), LS measurements by $p$-SWE failed. In particular, patients with failed $p$-SWE more frequently had advanced fibrosis ( $90.0 \%$ vs. $39.2 \%, \mathrm{P}=0.006$ ), and also had higher BMI (mean, $33.7 \pm 4.9 \mathrm{~kg} / \mathrm{m}^{2} ; \mathrm{P}=0.011$ ) and higher TE measurements (mean, $28.9 \pm 25.4 \mathrm{kPa} ; \mathrm{P}<0.001)$ than patients with valid $\mathrm{LS}$ measurements. Cassinotto et al. [12], along with other evidence, also reported fewer reliable results in patients with a BMI $\geq 30 \mathrm{~kg} / \mathrm{m}^{2}$ and a waist circumference $\geq 102 \mathrm{~cm}[16,24]$. We speculate that, in our study population, this non-negligible percentage of failed p-SWE could be related to US equipment technology. Of note, the US equipment is undergoing constant updates, and the latest version deems the results to be reliable when the RMI is $\geq 0.4$ instead of the previously suggested value of $\geq 0.6$. This technical aspect could potentially decrease the percentages of failed p-SWE measurements, and applying this cutoff would have allowed us to include many more patients in our study.

It should also be noted that, on the basis of the current literature, each ultrasound equipment is different from the others, with particular reference to quantitative evaluation and optimal SWE cutoff values to classify significant and advanced liver fibrosis, which are specific for each ultrasound machine. Indeed, different software methods can be used to measure shear wave arrival time and speed [25]. This could be considered a limitation of $p-S W E$, since a reliable follow-up should always be performed in the same patient using the same US machine. In our study, according to EFSUMB guidelines, we evaluated all patients using the same US equipment by the same manufacturer; as such, we report our experience with this specific US equipment. Therefore, cutoff values are different for other US machines, even if the p-SWE technique has been well-known for some years. It should be also noted that an unquestionable advantage of TE with respect to $\mathrm{p}$-SWE is the capability to quantify liver fatty content by using the controlled attenuation parameter (CAP), which measures ultrasound beam attenuation (a parameter directly related to the amount of steatosis), and enables the stratification of each patient in a different stage (no steatosis; mild, moderate, or severe steatosis) with a robust correlation with biopsy results. CAP measurements are important for the TE LS evaluation since $L S$ can be overestimated in patients with high CAP values, which has led to the adjustment of TE cutoff values and the development of new ultrasound software [26-28]. Moreover, before performing SWE, confounding factors should be assessed to prevent the overestimation of LS measurements; patients with liver inflammation (AST and/or ALT elevation $>5$ times the normal limits), obstructive cholestasis, liver congestion, acute hepatitis, and infiltrative liver diseases should be excluded before measuring $L S$, as recommended by EFSUMB guidelines [14].

Our study has multiple limitations that need to be acknowledged. First, our study population was limited by the relatively small number of prospectively-enrolled patients in a single Institution related to our selection criteria and the RMI cutoff values considered to indicate reliable measurements. The final number of included patients and the exclusion of patients with failed SWE 
measurements may have limited the evaluation of differences in diagnostic performance between TE and P-SWE with different measurements. However, all the included subjects underwent histopathological examinations with percutaneous liver biopsy within 1 month and underwent laboratory tests on the same day of TE and p-SWE evaluation. Second, p-SWE was performed by a single radiologist, therefore, inter-reader agreement could not be evaluated. Other studies have already investigated the inter- and intra-reader reproducibility of SWE techniques [29]. Finally, our population included only four patients lacking fibrosis (F0) on biopsy specimens.

In conclusion, TE and p-SWE had similar fair-to-good diagnostic performance for the diagnosis of biopsy-proven significant and advanced fibrosis in patients with NAFLD.

ORCID: Adele Taibbi: https://orcid.org/0000-0001-6442-744X; Salvatore Petta: https:// orcid.org/0000-0002-0822-9673; Domenica Matranga: https://orcid.org/0000-00022466-937X; Giovanni Caruana: https://orcid.org/0000-0002-0239-5005; Roberto Cannella: https://orcid.org/0000-0002-3808-0785; Vito Di Marco: https://orcid.org/00000001-6397-4206; Massimo Midiri: https://orcid.org/0000-0003-1824-7549; Tommaso Vincenzo Bartolotta: https://orcid.org/0000-0002-8808-379X

\section{Author Contributions}

Conceptualization: Taibbi A, Petta S, Di Marco V, Midiri M, Bartolotta TV. Data acquisition: Taibbi A, Petta S, Caruana G, Busè G, Bartolotta TV. Data analysis or interpretation: Taibbi A, Matranga D, Caruana G, Cannella R, Bartolotta TV. Drafting of the manuscript: Taibbi A, Cannella R. Critical revision of the manuscript: Taibbi A, Petta $S$, Matranga D, Caruana G, Cannella R, Busè G, Di Marco V, Midiri M, Bartolotta TV. Approval of the final version of the manuscript: all authors.

\section{Conflict of Interest}

Tommaso Vincenzo Bartolotta serves as Editor for the Ultrasonography, but has no role in the decision to publish this article. All remaining authors have declared no conflicts of interest.

\section{References}

1. Taibbi A, Picone D, Midiri M, La Grutta L, Bartolotta TV. Diffuse liver diseases: role of imaging. Semin Ultrasound CT MR 2018;39:193205.

2. Rinella ME. Nonalcoholic fatty liver disease: a systematic review. JAMA 2015;313:2263-2273.

3. Castera L, Friedrich-Rust M, Loomba R. Noninvasive assessment of liver disease in patients with nonalcoholic fatty liver disease. Gastroenterology 2019;156:1264-1281.
4. Williams CD, Stengel J, Asike MI, Torres DM, Shaw J, Contreras M, et al. Prevalence of nonalcoholic fatty liver disease and nonalcoholic steatohepatitis among a largely middle-aged population utilizing ultrasound and liver biopsy: a prospective study. Gastroenterology 2011;140:124-131.

5. Loomba R, Sanyal AJ. The global NAFLD epidemic. Nat Rev Gastroenterol Hepatol 2013;10:686-690.

6. Petta S, Maida M, Macaluso FS, Di Marco V, Camma C, Cabibi D, et al. The severity of steatosis influences liver stiffness measurement in patients with nonalcoholic fatty liver disease. Hepatology 2015;62:1101-1110.

7. Lee SM, Kim MJ, Yoon JH, Hong W, Ha HI, Lee K, et al. Comparison of point and 2-dimensional shear wave elastography for the evaluation of liver fibrosis. Ultrasonography 2020;39:288-297.

8. Bamber J, Cosgrove D, Dietrich CF, Fromageau J, Bojunga J, Calliada $F$, et al. EFSUMB guidelines and recommendations on the clinical use of ultrasound elastography. Part 1: Basic principles and technology. Ultraschall Med 2013;34:169-184.

9. Nierhoff J, Chavez Ortiz AA, Herrmann E, Zeuzem S, Friedrich-Rust $M$. The efficiency of acoustic radiation force impulse imaging for the staging of liver fibrosis: a meta-analysis. Eur Radiol 2013;23:30403053 .

10. Ferraioli G, Tinelli C, Dal Bello B, Zicchetti M, Filice G, Filice C, et al. Accuracy of real-time shear wave elastography for assessing liver fibrosis in chronic hepatitis C: a pilot study. Hepatology 2012;56:2125-2133.

11. Cassinotto C, Lapuyade B, Mouries A, Hiriart JB, Vergniol J, Gaye $D$, et al. Non-invasive assessment of liver fibrosis with impulse elastography: comparison of supersonic shear imaging with ARFI and FibroScan $\circledast$. J Hepatol 2014;61:550-557.

12. Cassinotto C, Boursier J, de Ledinghen V, Lebigot J, Lapuyade $B$, Cales $\mathrm{P}$, et al. Liver stiffness in nonalcoholic fatty liver disease: a comparison of supersonic shear imaging, FibroScan, and ARFI with liver biopsy. Hepatology 2016;63:1817-1827.

13. Liu H, Fu J, Hong R, Liu L, Li F. Acoustic radiation force impulse elastography for the non-invasive evaluation of hepatic fibrosis in non-alcoholic fatty liver disease patients: a systematic review and meta-analysis. PLoS One 2015;10:e0127782.

14. Dietrich CF, Bamber J, Berzigotti A, Bota S, Cantisani V, Castera L, et al. EFSUMB guidelines and recommendations on the clinical use of liver ultrasound elastography, update 2017 (long version). Ultraschall Med 2017;38:e16-e47.

15. Boursier J, Zarski JP, de Ledinghen V, Rousselet MC, Sturm N, Lebail $B$, et al. Determination of reliability criteria for liver stiffness evaluation by transient elastography. Hepatology 2013;57:11821191.

16. Kang HJ, Lee JY, Lee KB, Joo I, Suh KS, Lee HK, et al. Addition of reliability measurement index to point shear wave elastography: prospective validation via diagnostic performance and 
reproducibility. Ultrasound Med Biol 2019;45:1594-1602.

17. Leong WL, Lai LL, Nik Mustapha NR, Vijayananthan A, Rahmat K, Mahadeva S, et al. Comparing point shear wave elastography (ElastPQ) and transient elastography for diagnosis of fibrosis stage in non-alcoholic fatty liver disease. J Gastroenterol Hepatol 2020;35:135-141.

18. Kleiner DE, Brunt EM, Van Natta M, Behling C, Contos MJ, Cummings $\mathrm{OW}$, et al. Design and validation of a histological scoring system for nonalcoholic fatty liver disease. Hepatology 2005;41:1313-1321.

19. Vernon G, Baranova A, Younossi ZM. Systematic review: the epidemiology and natural history of non-alcoholic fatty liver disease and non-alcoholic steatohepatitis in adults. Aliment Pharmacol Ther 2011;34:274-285.

20. Furlan A, Tublin ME, Yu L, Chopra KB, Lippello A, Behari J. Comparison of $2 D$ shear wave elastography, transient elastography, and MR elastography for the diagnosis of fibrosis in patients with nonalcoholic fatty liver disease. AJR Am J Roentgenol 2020;214:W20-W26.

21. Jiang $W$, Huang $S$, Teng $H$, Wang $P, W u ~ M$, Zhou $X$, et al. Diagnostic accuracy of point shear wave elastography and transient elastography for staging hepatic fibrosis in patients with non-alcoholic fatty liver disease: a meta-analysis. BMJ Open 2018;8:e021787.

22. Roccarina D, logna Prat L, Buzzetti E, Guerrero Misas M, Arico FM, Saffioti $F$, et al. Establishing reliability criteria for liver ElastPQ shear wave elastography (ElastPQ-SWE): comparison between 10, 5 and 3 measurements. Ultraschall Med 2021;42:204-213.

23. Fang C, Jaffer OS, Yusuf GT, Konstantatou E, Quinlan DJ, Agarwal K, et al. Reducing the number of measurements in liver point shearwave elastography: factors that influence the number and reliability of measurements in assessment of liver fibrosis in clinical practice. Radiology 2018;287:844-852.

24. Mikolasevic I, Orlic L, Franjic N, Hauser G, Stimac D, Milic S. Transient elastography (FibroScan $(囚)$ ) with controlled attenuation parameter in the assessment of liver steatosis and fibrosis in patients with nonalcoholic fatty liver disease: where do we stand? World J Gastroenterol 2016;22:7236-7251.

25. Sugimoto K, Moriyasu F, Oshiro H, Takeuchi H, Yoshimasu Y, Kasai Y, et al. Clinical utilization of shear wave dispersion imaging in diffuse liver disease. Ultrasonography 2020;39:3-10.

26. Petta S, Wong VW, Camma C, Hiriart JB, Wong GL, Marra F, et al. Improved noninvasive prediction of liver fibrosis by liver stiffness measurement in patients with nonalcoholic fatty liver disease accounting for controlled attenuation parameter values. Hepatology 2017;65:1145-1155.

27. Sasso M, Miette V, Sandrin L, Beaugrand M. The controlled attenuation parameter (CAP): a novel tool for the non-invasive evaluation of steatosis using Fibroscan. Clin Res Hepatol Gastroenterol 2012;36:13-20.

28. Ferraioli G, Soares Monteiro LB. Ultrasound-based techniques for the diagnosis of liver steatosis. World J Gastroenterol 2019;25:6053-6062.

29. Yoo J, Lee JM, Joo I, Yoon JH. Assessment of liver fibrosis using 2-dimensional shear wave elastography: a prospective study of intra- and inter-observer repeatability and comparison with point shear wave elastography. Ultrasonography 2020;39:52-59. 